\title{
Evaluación del Uso de Melaza en Dietas Para Cerdos en Crecimiento y Engorde
}

\author{
Johanna Elizabeth Escobar Carvajal, Melina Damaris Macías Mock, Rogel Castillo, Miguel Vélez
}

\begin{abstract}
Resumen. En la producción moderna de cerdos, la alimentación representa del 70 a 80\% de los costos. En zonas tropicales que no tienen ventajas comparativas para producir cereales, pueden utilizar alternativas alimenticias como papa, yuca, malanga, camote, caña de azúcar, melaza y otros subproductos agro-industriales. El objetivo de este estudio fue evaluar el efecto de cuatro niveles de melaza en la dieta de cerdos en las etapas de crecimiento y engorde. El estudio se realizó entre enero y mayo de 2005 en la Escuela Agrícola Panamericana, Honduras. Se utilizaron cuatro grupos de 30 cerdos cada uno, con peso promedio de $27 \mathrm{~kg}$ en la etapa de crecimiento y de $48 \mathrm{~kg}$ en la etapa de engorde, los cerdos eran cruces de Landrace, Yorkshire, Duroc y cruce con verracos híbridos de la empresa PIC. Los tratamientos fueron 0, 10, 20 ó 30\% de melaza en la dieta para ambas etapas. Se utilizó un Diseño Completamente al Azar, con cuatro tratamientos y tres repeticiones. Se realizó un Análisis de Varianza mediante el Modelo Lineal General (GLM), con una probabilidad menor a 0.05. Los cerdos que recibieron la dieta con $0 \%$ de melaza tuvieron una mayor ganancia de peso (781 g/cerdo/día) que los que recibieron melaza, no se encontraron diferencias entre los tratamientos en el consumo de alimento ni en el índice de conversión alimenticia en la etapa de crecimiento. En la etapa de engorde no se encontraron diferencias significativas ( $\mathrm{P}>$ 0.05) para ninguna de las variables evaluadas. Es posible alimentar cerdos con dietas que contengan hasta un $30 \%$ de melaza sin alterar el rendimiento en canal, grasa dorsal ni área del lomo. Los costos de alimentación se pueden reducir hasta un 22\% en dietas con 30\% de melaza para la etapa de crecimiento y $24 \%$ en la etapa de engorde, sin embargo, el mayor margen sobre el costo de alimentación encontrado fue en dietas con $20 \%$.
\end{abstract}

Palabras clave: Área de lomo, desempeño animal, grasa dorsal, rendimiento de canal

Abstract. In swine production, feed represents 70 to $80 \%$ of the total cost. In the tropics, the production of corn and soybean, the main ingredients in the swine's diet, is very low. This is a disadvantage compared with farmers in the United States, where there are alternatives to replace these ingredients with other sources of feed like potato, cassava, sweet potato, sugar cane, molasses and other by-products of the agro industry. The objective of the present work was to evaluate the effect of four levels of molasses in the growing and finishing diets of pigs. The study was conducted between January and May, 2005, in the Escuela Agrícola Panamericana, Zamorano, Honduras. A total of 120 pigs were allotted to four treatment groups averaging $27 \mathrm{~kg}$ initial weight for the growing period, and $48 \mathrm{~kg}$ for the finishing diet. The pigs were crossbred of Landrace, Yorkshire, Duroc and PIC hybrids. The treatments were 0, 10, 20 or 30\% of molasses in the diets. The experimental design was a Completely Randomize Design, with four treatments and three replicates. An Analysis of Variances was used with the General Lineal Model (GLM), with a 0.05 significance level. The pigs with $0 \%$ of molasses had a better average daily gain (ADG) (781 g/day) than pigs fed with the other diets. There was no difference between treatments for feed intake or the feed conversion ratio in the growing phase. There were no differences $(\mathrm{P}>0.05)$ for the variables in the finishing phase. Our results indicate that it is possible to feed pigs with up to $30 \%$ of molasses in the diet. The feed costs can be reduced $30 \%$ and $24 \%$ for the growing and finishing diets, respectively. However, the best margin over feed cost was for the diet with $20 \%$ molasses.

Key words: Animal performance, backfat, carcass weight, loin area.

\section{Introducción}

Las proyecciones de la producción mundial de carnes para el 2005 señalan un crecimiento cercano a $1 \%$, con lo que se alcanzaría un volumen de 253.6 millones de toneladas. Para la carne de cerdo se proyecta un crecimiento de $1.6 \%$, lo que significa una producción cercana a 97.7 millones de toneladas. La carne de cerdo es la que más se produce en el mundo, y representa el 38.5\% en el volumen total (FAO 2004).

La disponibilidad de materias primas y alimentos balanceados, así como la posibilidad de cruce con razas más magras y de mayor crecimiento, ha traído consigo una evolución en la producción del cerdo. El 
animal ya no depende de la producción natural para su alimentación y el ganadero aporta alimentos adecuados a cada tipo de animal, en cada fase de su vida (gestación, lactación, cría y engorde); de este modo consigue que el cerdo alcance el peso óptimo de sacrifico en menos tiempo (De Pedro y García 2002).

En la producción moderna de cerdos, la alimentación representa del 70 al $80 \%$ de los costos. En países desarrollados de clima templado, la porción energética de la alimentación está basada en cereales como el maíz, la cebada y el sorgo. En zonas tropicales que no tienen ventajas comparativas para producir cereales, se recomienda utilizar alimentos que estén disponibles en el trópico tales como papa, yuca, malanga, camote, caña de azúcar y algunos subproductos agro-industriales (Sarria et al. 1990).

Por razones económicas, el azúcar crudo ha sido poco utilizado para la alimentación de cerdos y son pocos los informes técnicos sobre su uso; sin embargo, los resultados biológicos han sido muy positivos al comparar dietas con altos niveles de azúcar con las dietas tradicionales que utilizan el maíz como fuente energética. Ya que el azúcar carece de proteína y su contenido de vitaminas y minerales es bajo, el éxito de su utilización dependerá de una suplementación adecuada (Zapata 2000; Pérez et al. 1991).

La clarificación, concentración y cristalización del jugo de caña en el ingenio azucarero origina las mieles. En el flujo tecnológico se producen cuatro tipos de mieles: la meladura o primera miel (miel rica cuando se hidroliza para evitar la cristalización de sacarosa), la miel A que se produce cuando se extrae el $75 \%$ del total de azúcar recuperable, la miel B cuando se completa el $86 \%$ de cristalización y por último la miel final o melaza cuando ya no es posible la obtención de la sacarosa (Figueroa 1989).

La melaza es un concentrado de hidratos de carbonos y los azúcares representan un alto porcentaje de su materia seca. Es un producto apetecible por las especies animales y al ser añadido a la dieta en niveles bajos (5-10\%), incrementa su palatabilidad y reduce pérdidas por polvo; sin embargo, en países productores de azúcar la usan en niveles superiores (Poballe 2004).

Considerando lo anterior, se hizo un estudio con el objetivo evaluar el efecto de cuatro niveles de melaza en la dieta de cerdos de crecimiento y engorde sobre la ganancia de peso, consumo de alimento, índice de conversión alimenticia, rendimiento en canal caliente, grasa dorsal y área de lomo, así como el efecto en los costos de producción.

\section{Materiales y Métodos}

El estudio se realizó entre enero y mayo de 2005 en la unidad de cerdos de la Escuela Agrícola Panamericana. Esta unidad está ubicada a $30 \mathrm{~km}$ al SE de Tegucigalpa, a una altura de $800 \mathrm{msnm}$, temperatura promedio de $24^{\circ} \mathrm{C}$ y una precipitación anual de $1100 \mathrm{~mm}$.

Se utilizaron cuatro grupos de 30 cerdos cada uno, con peso promedio de $27 \mathrm{~kg}$ en la etapa de crecimiento y de $48 \mathrm{~kg}$ en la etapa de engorde. Los cerdos eran cruces de Landrace, Yorkshire, Duroc y cruce con verracos híbridos de la empresa PIC.

Se utilizaron cuatro tratamientos: 0, 10, 20 y 30\% de melaza en la dieta (Cuadro 1), y se balancearon según los requerimientos del National Research Council (NRC 1998). La etapa de crecimiento duró seis semanas y la de engorde siete semanas hasta obtener un peso final promedio de $85 \mathrm{~kg}$.

Los cerdos se ubicaron en 12 corrales con pisos de cemento y comederos de tubo de PVC, considerando cada corral una unidad experimental (tres repeticiones por tratamiento). La alimentación fue a libre acceso, las dietas con 0 y 10\% de melaza venían listas de la planta de concentrados, mientras que en las dietas de 20 y $30 \%$ el alimento seco se mezcló con melaza en el sitio antes de ofrecerlo a los cerdos. Este proceso se debió a que la maquinaria en la planta no tiene la capacidad de mezclar alimentos con alto porcentaje de melaza, debido a que se forma un concentrado muy denso, lo que dificulta su salida, transporte y almacenamiento.

Al finalizar la etapa de engorde los cerdos se sacrificaron en la Planta de Cárnicos de Zamorano; se evaluó el peso en canal caliente, rendimiento en canal y grosor de la grasa dorsal. Las variables medidas fueron: ganancia diaria de peso (GDP), consumo de alimento (CA), índice de conversión alimenticia (ICA), rendimiento en canal caliente, espesor de grasa dorsal y se realizó un análisis marginal en el que se consideró los costos de alimentación. 
Cuadro No.1 Composición de las dietas utilizadas en las etapas de crecimiento y engorde de los cerdos.

\begin{tabular}{|c|c|c|c|c|c|c|c|c|c|}
\hline \multirow[b]{2}{*}{ Ingredientes } & \multirow{2}{*}{$\begin{array}{l}\text { Costo } \\
\text { L./kg }\end{array}$} & \multicolumn{4}{|c|}{ Dieta de Crecimiento } & \multicolumn{4}{|c|}{ Dieta de Engorde } \\
\hline & & \multicolumn{8}{|c|}{ Porcentaje de inclusión } \\
\hline Maíz & 3.7 & 71.9 & 57.4 & 44.0 & 31.5 & 67.1 & 54.5 & 41.2 & 27.9 \\
\hline Aceite & 9.0 & 1.5 & 3.5 & 4.5 & 5.0 & 2.0 & 4.5 & 5.0 & 6.0 \\
\hline Semolina de arroz & 3.1 & & & & & 20.0 & 15.0 & 15.0 & 15.0 \\
\hline Harina Carne & 5.4 & 3.0 & 3.0 & 4.0 & 5.0 & 2.0 & 3.0 & 3.0 & 3.0 \\
\hline Harina Camarón & 5.9 & 10.0 & 10.0 & 10.0 & 10.7 & 0.0 & 9.0 & 9.0 & 9.0 \\
\hline Harina Soya & 7.3 & 13.0 & 15.5 & 17.0 & 18.0 & 7.0 & 3.8 & 6.2 & 8.5 \\
\hline Carbonato de calcio & 0.6 & & & & & 0.8 & & & \\
\hline Lisina & 71.6 & & & & & 0.3 & 0.1 & 0.1 & 0.0 \\
\hline Sal Común & 1.4 & 0.2 & 0.2 & 0.2 & 0.2 & 0.5 & 0.2 & 0.2 & 0.2 \\
\hline Vitamina Cerdos & 28.0 & 0.3 & 0.3 & 0.3 & 0.3 & 0.3 & 0.3 & 0.3 & 0.3 \\
\hline Melaza & 1.0 & 0.0 & 10.0 & 20.0 & 30.0 & 0.0 & 10.0 & 20.0 & 30.0 \\
\hline Tylan & 270.0 & 0.1 & 0.1 & 0.1 & 0.1 & 0.1 & 0.1 & 0.1 & 0.1 \\
\hline Total & & 100.0 & 100.0 & 100.0 & 100.0 & 100.0 & 100.0 & 100.0 & 100.0 \\
\hline Costo/kg & & 4.7 & 4.5 & 4.4 & 4.2 & 4.3 & 4.2 & 4.0 & 3.9 \\
\hline
\end{tabular}

Tasa de Cambio 18.86 L./\$

Los cerdos se pesaron al inicio del ensayo y al final de cada fase de alimentación. Se pesó el alimento ofrecido diariamente y el rechazado al final de cada fase de alimentación. El ICA se evaluó mediante la división del alimento ofrecido entre la ganancia de peso, en cada fase de alimentación.

Se pesaron las canales dos horas después del sacrificio, el espesor de grasa dorsal se midió en la décima costilla, 24 horas después del sacrificio, con un pie de rey. El rendimiento en canal se hizo comparando el peso vivo del animal con el peso en canal (sin patas, vísceras ni cabeza). Para el análisis de costos sólo se consideraron los costos de la alimentación.

Se utilizó un DCA (diseño completamente al azar) con cuatro tratamientos y tres repeticiones por tratamiento. Se realizó un Análisis de Varianza (ANDEVA) mediante el Modelo Lineal General (GLM) y una separación de medias por el método de análisis SNK, del paquete estadístico Statistic Analysis System $\left(\right.$ SAS $^{\circledR}$ 2003). Para la variable rendimiento en canal caliente se corrigieron los valores con la función arcoseno.

\section{Resultados y Discusión}

\section{Etapa de Crecimiento}

Ganancia diaria de peso. Los cerdos alimentados sin melaza tuvieron mayor ganancia de peso $(\mathrm{P}=0.03)$ que los que recibieron melaza (Cuadro 2); entre los que recibieron melaza fueron similares $(\mathrm{P}>0.05)$. Estas ganancias de peso son mayores a las reportadas por González et al. (1993) en Cuba; ellos reportaron 562 y 529 g/día con dietas de: miel final o melaza más concentrado con alto porcentaje de proteína y miel final a voluntad más concentrado con alto porcentaje de proteína con 35.6\% de sustitución del cereal por saccharina de bagacillo.

La GDP al añadir melaza fue menor que las encontradas por Díaz et al. (2000) en Cuba, quienes obtuvieron un promedio de 677 g/día, en cerdos con melaza ad libitum. Esto se debe a que el peso inicial en este experimento fueron menores a los usados por Díaz et al., quienes concluyeron que a mayor peso de los cerdos al inicio de los tratamientos, mayor será su ganancia de peso. También fue menor a las 
encontradas por Babatunde et al. (1975) utilizando dietas con melaza hasta un $40 \%$, obtuvieron mayores GDP que los tratamientos con melaza, reportando un promedio de $668 \mathrm{~g} /$ día, en cerdos con un peso inicial de $17.13 \mathrm{~kg}$ y peso final de $64.7 \mathrm{~kg}$.

Consumo diario de alimento. No se encontraron diferencias $(\mathrm{P}>0.05)$ en el consumo de alimento (Cuadro 2). Dichos consumos son menores que los reportados por Díaz et al. (2000) de 2,543 g en cerdos alimentados con base en concentrado y melaza ad libitum. Díaz y Rodríguez (2002) reportaron un consumo promedio de $1,985 \mathrm{~g}$, estos resultados son mayores a los obtenidos en este experimento que en promedio fueron de 1,762 g. Estas diferencias se deben a la forma de suministrar el alimento, estos autores utilizaron recipientes exclusivamente para el azúcar crudo y la miel final, lo que estimuló un mayor consumo de este alimento.

Índice de conversión alimenticia. El ICA no mostró diferencias $(\mathrm{P}>0.05)$ entre los tratamientos (Cuadro 1$).$ Estos índices son mejores que los reportados por González et al. (1993) de 5.29 y 5.97 en su experimento con dietas que contenían miel final y a los de Díaz et al. (2000) cuyo ICA fue de 3.45.

\section{Etapa de Engorde}

Ganancia diaria de peso. No se encontró diferencia significativa entre tratamientos. Sin embargo, la GDP promedio de 793 g (Cuadro 3) fue mayor que la reportada por Díaz et al. (2000) quienes encontraron una GDP promedio de $746 \mathrm{~g}$ en cerdos de engorde alimentados con concentrado y melaza ad libitum, debido probablemente a los altos consumos de melaza reportados por estos autores.

Las ganancias diarias de peso son menores a las obtenidas por Rosales (2004), de 972.9 g con la dieta control sin Paylean con 6\% de melaza, en cerdos con peso promedio inicial de $72.7 \mathrm{~kg}$ y peso promedio a sacrificio de $101.9 \mathrm{~kg}$, debido probablemente al menor contenido de melaza de la dieta y al mayor peso inicial de los cerdos en dicho experimento.

Los índices obtenidos en este estudio son menores a los reportados por Piña (1988), que obtuvo una ganancia diaria de peso de 840 g con un $40 \%$ de melaza sustituyendo al maíz, esto se debe a un mayor consumo de alimento obtenida por este autor, que permite de igual manera una mejor GDP.

Consumo diario de alimento. El consumo diario de alimento fue de $2,750 \mathrm{~g} /$ día para el grupo con un peso inicial de $48.45 \mathrm{~kg}$ (Cuadro 3) que es mayor a los consumos obtenidos por Díaz et al. (2000) de 2,340 y 2,480 g para los grupos cuyo peso vivo inicial fue 19 y $29 \mathrm{~kg}$, respectivamente.

Piña (1988) reportó un consumo de 3700 g en cerdos suplementados con $40 \%$ de melaza, mayor al obtenido en este experimento, esto se debe a que la densidad energética de la dieta se ve afectada por el aporte de energía de la melaza que es menor al de otras fuentes como maíz, por lo que al aumentar la cantidad de melaza en la dieta tiende a incrementar el consumo para cubrir los requerimientos energéticos.

Índice de Conversión Alimenticia. Los índices obtenidos no mostraron diferencias $(\mathrm{P}>0.05)$ entre tratamientos (Cuadro 3), y fueron muy similares a los obtenidos por Díaz et al. (2000) de 3.45, 3.27 y 3.15 en su experimento en el que muestra una mejora en la eficiencia del concentrado al aumentar el peso vivo inicial en el engorde.

La conversión alimenticia en las dietas con 0,10 y 30\% fue menor que la obtenida por Rosales (2004), quien reportó 3.5 en cerdos con $72.7 \mathrm{~kg}$ de peso inicial con una dieta a base de maíz y harina de soya.

Piña (1988) encontró un ICA de 3.86 y de 4.40 para el grupo testigo con maíz y el suplementado con $40 \%$ de melaza, respectivamente, los que son mayores a los obtenidos en el experimento, esto se debe a que la densidad energética del concentrado y su calidad disminuye con porcentajes altos de melaza por lo que es necesario un mayor consumo.

Características de Composición de la canal. Para los principales indicadores de rendimiento y de calidad de la canal no se encontraron diferencias $(\mathrm{P}>0.05)$ para ninguna de las variables (Cuadro 4), debido a que todas las dietas se formularon para cumplir con los requerimientos de nutrientes de los cerdos. 
Cuadro 2. Desempeño de los cerdos en la etapa de crecimiento con cuatro niveles de melaza en la dieta.

\begin{tabular}{|c|c|c|c|c|c|}
\hline \multirow{2}{*}{ Melaza (\%) } & \multicolumn{2}{|c|}{ g/cerdo/día } & \multicolumn{2}{|c|}{ Peso (kg) } \\
\cline { 2 - 3 } \cline { 5 - 5 } & GDP & CA & ICA & Inicial & Final \\
\hline 0 & $781 \pm 30^{\mathrm{a}}$ & $1,976 \pm 567^{\mathrm{a}}$ & $2.5 \pm 0.7^{\mathrm{a}}$ & 29.2 & 62.5 \\
\hline 10 & $581 \pm 27^{\mathrm{b}}$ & $1,631 \pm 281^{\mathrm{a}}$ & $2.8 \pm 0.6^{\mathrm{a}}$ & 27.0 & 51.4 \\
\hline 20 & $578 \pm 90^{\mathrm{b}}$ & $1,700 \pm 86^{\mathrm{a}}$ & $3.0 \pm 0.3^{\mathrm{a}}$ & 27.1 & 51.4 \\
\hline 30 & $598 \pm 46^{\mathrm{b}}$ & $1,743 \pm 160^{\mathrm{a}}$ & $2.9 \pm 0.0^{\mathrm{a}}$ & 26.4 & 51.5 \\
\hline
\end{tabular}

Promedios con letras diferentes en la misma columna son diferentes $(\mathrm{P}<0.05)$

GDP: Ganancia diaria de peso, CA: Consumo de alimento, ICA: Índice de conversión alimenticia (kilogramo de alimento por kilogramo de peso vivo ganado).

Cuadro 3. Desempeño de los cerdos en la etapa de engorde con cuatro niveles de melaza en la dieta.

\begin{tabular}{|c|c|c|c|c|c|}
\hline \multirow{2}{*}{ Melaza (\%) } & \multicolumn{2}{|c|}{ g/cerdo/día } & \multicolumn{2}{|c|}{ Peso (kg) } \\
\cline { 2 - 3 } \cline { 5 - 6 } & GDP & CA & ICA & Inicial & Final \\
\hline 0 & $848 \pm 95$ & $2,819 \pm 414$ & $3.3 \pm 0.4$ & 46.5 & 87.0 \\
\hline 10 & $705 \pm 87$ & $2,657 \pm 135$ & $3.8 \pm 0.4$ & 50.4 & 80.0 \\
\hline 20 & $828 \pm 76$ & $2,827 \pm 374$ & $3.4 \pm 0.2$ & 47.7 & 82.5 \\
\hline 30 & $790 \pm 17$ & $2,695 \pm 287$ & $3.4 \pm 0.3$ & 49.2 & 82.3 \\
\hline
\end{tabular}

GDP: Ganancia diaria de peso, CA: Consumo de alimento, ICA: Índice de conversión alimenticia.

Rendimiento de la canal caliente. Los rendimientos obtenidos fueron muy similares a los que normalmente se obtienen en la industria (Cuadro 4). El rendimiento en canal es de aproximadamente $70 \%$ y un $22 \%$ de subproductos. Rosales (2004) obtuvo un rendimiento de $69.2 \%$ para el grupo control con $6 \%$ de melaza en la dieta, este rendimiento fue ligeramente mayor al obtenido en este experimento que fue $68.4 \%$.

Espesor de la grasa dorsal y área del lomo (GD y AL). El espesor de grasa dorsal y área de lomo reportados por Rosales (2004) fueron de $1.82 \mathrm{~cm} \mathrm{y}$ $45.2 \mathrm{~cm}^{2}$, respectivamente en cerdos con un peso final de $101.9 \mathrm{~kg}$, estos parámetros son superiores a los obtenidos en este experimento (Cuadro 4), por el mayor peso final y tamaño de los animales. A pesar del alto contenido de melaza en la dieta, la grasa dorsal se mantuvo dentro de los parámetros de la industria, con dietas con bajo contenido de melaza.

Costos. El costo del alimento bajó al aumentar el contenido de melaza (Cuadros 5 y 6). En la etapa de crecimiento el uso de $30 \%$ de melaza tuvo el menor costo, debido a que la cantidad de alimento consumido y el ICA fueron menores. En la etapa de engorde la dieta con $30 \%$ de melaza tuvo el menor costo de alimentación sin alterar la ganancia de peso ni el ICA.

Cuadro 4. Rendimiento y características de la canal de cerdos alimentados con cuatro niveles de melaza.

\begin{tabular}{|c|l|l|c|}
\hline $\begin{array}{c}\text { Melaza } \\
(\%)\end{array}$ & $\begin{array}{c}\text { Rendimiento } \\
\text { en canal (\%) }\end{array}$ & $\begin{array}{c}\text { Espesor de } \\
\text { grasa dorsal } \\
(\mathrm{cm})\end{array}$ & $\begin{array}{c}\text { Área del } \\
\text { lomo } \\
\left(\mathrm{cm}^{2}\right)\end{array}$ \\
\hline 0 & $66.6 \pm 2.5$ & $1.6 \pm 0.4$ & $35.9 \pm 4.7$ \\
\hline 10 & $69.1 \pm 7.0$ & $1.7 \pm 0.5$ & $42.7 \pm 6.9$ \\
\hline 20 & $70.6 \pm 9.4$ & $1.5 \pm 0.6$ & $40.5 \pm 8.1$ \\
\hline 30 & $67.5 \pm 4.6$ & $1.6 \pm 0.5$ & $38.9 \pm 8.7$ \\
\hline
\end{tabular}

El costo total para ambas fases fue menor para la dieta con 30\% de melaza (Cuadro 6). El mayor margen sobre el costo se obtuvo con $20 \%$ de melaza, a pesar de que el costo de alimentación fue más alto que el de las otras dietas con melaza (Cuadro 7), esto se debe a que los cerdos de este tratamiento lograron un mayor rendimiento en canal. El precio por $\mathrm{kg}$ de peso vivo fue de L 36.96. 
Cuadro 5. Costos de alimentación de los cerdos en la etapa de crecimiento.

\begin{tabular}{|c|c|c|c|c|c|c|c|}
\hline Melaza (\%) & \multicolumn{2}{|c|}{ Peso (kg) } & GPT (kg) & ICA & \multicolumn{2}{c|}{ CTA } & \multicolumn{2}{c|}{ Costo } \\
\hline & Inicial & Final & & & $\mathrm{kg}$ & $\mathrm{L} . / \mathrm{kg}$ & L./cerdo \\
\hline 0 & 29.5 & 62.5 & 33.0 & 2.5 & 82.5 & 4.7 & 387.8 \\
\hline 10 & 26.9 & 51.3 & 24.4 & 2.8 & 68.3 & 4.5 & 307.4 \\
\hline 20 & 27.0 & 51.3 & 24.3 & 3.0 & 72.9 & 4.4 & 320.8 \\
\hline 30 & 26.4 & 51.4 & 25.0 & 2.9 & 72.5 & 4.2 & 304.5 \\
\hline
\end{tabular}

Tasa de Cambio 18.86 L./\$

GPT: Ganancia de peso total

ICA: Índice de conversión alimenticia

CTA: Consumo total de alimento

Cuadro 6. Costos de alimentación de los cerdos en la etapa de engorde.

\begin{tabular}{|c|c|c|c|c|c|c|c|}
\hline Melaza (\%) & \multicolumn{2}{|c|}{ Peso (kg) } & GPT (kg) & ICA & CTA & \multicolumn{2}{c|}{ Costo } \\
\hline & Inicial & Final & & & $\mathrm{kg}$ & $\mathrm{L} . / \mathrm{kg}$ & L./cerdo \\
\hline 0 & 46.5 & 87.0 & 40.5 & 3.3 & 133.7 & 4.3 & 574.7 \\
\hline 10 & 50.4 & 80.0 & 29.6 & 3.8 & 112.5 & 4.2 & 472.4 \\
\hline 20 & 47.7 & 82.5 & 34.8 & 3.4 & 118.3 & 4.0 & 473.3 \\
\hline 30 & 49.2 & 82.3 & 33.1 & 3.4 & 112.5 & 3.9 & 438.9 \\
\hline
\end{tabular}

Tasa de Cambio 18.86 L./\$

GPT: Ganancia de peso total

ICA: Índice de conversión alimenticia

CTA: Consumo total de alimento

Cuadro 7. Análisis marginal de los costos de alimentación de los cerdos.

\begin{tabular}{|c|c|c|c|c|c|c|c|}
\hline \multirow{2}{*}{$\begin{array}{c}\text { Melaza } \\
(\%)\end{array}$} & \multicolumn{3}{|c|}{$\begin{array}{c}\text { Costo de alimento } \\
\text { (Lempiras/cerdo) }\end{array}$} & \multirow{2}{*}{$\begin{array}{c}\text { Costo total } \\
\text { (Lempiras/cerdo) }\end{array}$} & $\begin{array}{c}\text { Peso final } \\
(\mathrm{kg})\end{array}$ & Ingreso & Margen \\
\cline { 2 - 3 } & Inicio & Crecimiento & Engorde & & & \multicolumn{2}{|c|}{ Lempiras/cerdo } \\
\hline 0 & 247.0 & 390.7 & 574.7 & $1,212.4$ & 87.0 & $2,141.5$ & 929.2 \\
\hline 10 & 247.0 & 309.6 & 472.4 & $1,029.0$ & 80.0 & $2,043.2$ & $1,014.2$ \\
\hline 20 & 247.0 & 316.5 & 473.3 & $1,036.8$ & 82.5 & $2,152.7$ & $1,115.9$ \\
\hline 30 & 247.0 & 306.6 & 438.9 & 992.5 & 82.3 & $2,053.2$ & $1,060.7$ \\
\hline
\end{tabular}

Tasa de Cambio 18.86 L./\$ 


\section{Conclusiones}

En la etapa de crecimiento el uso de $0 \%$ de melaza proporcionó una mayor ganancia diaria de peso.

Bajo condiciones de Zamorano el uso de dietas con melaza al 10, 20 y $30 \%$ no afecta la ganancia diaria de peso, el consumo de alimento y el índice de conversión alimenticia en la etapa de engorde.

Es posible alimentar cerdos con dietas que contengan hasta un 30\% de melaza en la dieta sin alterar el rendimiento en canal caliente, grasa dorsal y área de lomo.

El costo de alimentación se puede reducir hasta un $22 \%$ en la etapa de crecimiento y un $24 \%$ en la de engorde, con dietas con 30\% de melaza. Sin embargo, el mayor margen sobre el costo de alimentación encontrado fue con dietas con $20 \%$ de melaza.

\section{Literatura Citada}

Asociación azucarera, El Salvador. 2004. Servicios y productos. Consultado: 19 agosto 2005. Disponible en: http://www.asociacionazucarera.com/productos.asp

Babatunde, G. M; Fetuga, B. L; Oyenuga, V. A. 1975. Effects of feeding graded levels of cane mollasses on the performance and carcass characteristics and organ weights of Yorkshire pigs in tropical environment. J. Anim. Sci. 40:632-639.

De Pedro E.; García J. 2002. Canales y Productos del Cerdo Ibérico: Aspectos productivos que afectan la calidad. Consultado el 13 de noviembre 2004. Disponible en: http://www.agroinformacion.com/leerarticulo.aspx?not $=307$

Díaz, C. P.; González, E.; Rodríguez, Y. 2000. Peso vivo inicial de cerdos en ceba y su relación con el comportamiento productivo en un sistema de alimentación con pienso y miel final ad libitum. Revista Cubana de Ciencias Agrícolas 34: 337-340.

Díaz, C. P.; Rodríguez, Y. 2002. Consumo voluntario de cerdos en crecimiento alimentados con azúcar crudo y miel final. Revista Cubana de Ciencias Agrícolas 36:161.
FAO, 2004. Estrategia para el fomento del sector cárnico. Consultado: 4 de noviembre 2004. Disponible en: http://www.fao.org/docrep/meeting/008/J2189s.htm\#P $\underline{34 \_2386}$

Figueroa, V. 1989. Experiencias cubanas en el uso de las mieles de caña para la alimentación porcina. Consultado: 19 agosto 2005. Disponible en: http://www.cipav.org.co/lrrd/lrrd1/1/vilda.htm

González, J.; Lezcano, P.; Castañeda, S. 1993. Sistema de alimentación para cerdos en ceba con dietas basadas en miel final y pienso con Saccharina de bagacillo. Revista Cubana de Ciencias Agrícolas. 27:185-192.

National Research Council (NRC), 1998. Nutrient requirement of Swine. Consultado 30 octubre 2004. Disponible en: http://www.nap.edu

Pérez, A.; González, J.; Domínguez, P.; Figueroa, V. 1991. Efecto de la suplementación en cerdos alimentados con miel "B" y desperdicios procesados. Habana, Cuba. Consultado: 2 octubre 2004. Disponible en:

http://www.zoetecnocampo.com/jump/jump.cgi?www. cipav.org.co/lrrd//rrd3/3/cuba.htm

Piña, A. M. 1988. La integración de la producción animal en la empresa azucarera comercial. Santo Domingo, República Dominicana. Consultado: 04 septiembre $2005 . \quad$ Disponible en: http://www.fao.org/docrep/003/s8850e/S8850E00.htm \#TOC

Poballe. 2004. Melaza de caña. Consultado: 30 agosto 2004. Disponible en: http://www.poballe.com/fichas/ficha10.htm

Rosales, E. 2004. Efectos de Paylean ${ }^{\circledR}$ sobre el desempeño productivo y la calidad de la carne de cerdo. Tesis al título de Ingeniera en Agroindustria en el Grado Académico de Licenciatura. El Zamorano, Honduras. $20 \mathrm{p}$.

SAS 2003. User guide: Statics SAS Inst; Inc; Cary, NC

Sarria, P., Solano, A. y Preston T. R. 1990. Utilización de jugo de caña y cachaza panalera en la alimentación de cerdos. Livestock Research for Rural Development. 2:92-101.

Vélez, M; Hincapié, J; Matamoros, I; Santillán, R. 2002. Producción de ganado lechero en el trópico. Ed. por Miguel Vélez. 4 ed. El Zamorano, Honduras, Zamorano Academic Press. 320 p.

Zapata, A.; 2000. Utilización de la caña de azúcar y sus derivados en la alimentación porcina. Editorial Scripto Ltda. Colombia. 153 p. 